JANUARY, 1972

\title{
TEMPERATURE OF A NONLINEARLY RADIATING SEMI-INFINITE SOLID*
}

\author{
BY JOSEPH B. KELLER (New York University, University Heights, and Courant Institute) \\ AND \\ W. E. OLMSTEAD (Northwestern University)
}

1. Introduction. Let $T(x, t)$ be the temperature of a semi-infinite heat-conducting solid occupying the half-space $x \geq 0$. We suppose that its surface radiates energy at a rate proportional to $[T(0, r)]^{n}$ and that the surface is heated by a source at a rate proportional to a given function $f(t)$. Here $n$ is a positive constant, the value $n=1$ corresponding to Newton's law of cooling and $n=4$ to Stefan's radiation law. If $T=0$ initially, then for $t>0, T$ is determined by the following initial boundary value problem:

$$
\begin{array}{ll}
T_{t}(x, t)=T_{x x}(x, t), & x>0, \quad t>0, \\
T_{x}(0, t)=\alpha T^{n}(0, t)-f(t), & t>0, \\
T^{\prime}(x, 0)=0, & x \geq 0, \\
T \rightarrow 0 \text { as } x \rightarrow \infty, & t \geq 0 .
\end{array}
$$

Here $\alpha>0$ is a given constant.

This problem has been considered by Mann and Wolf [1], Roberts and Mann [2] and Padmavally [3], while Friedman [4] has considered more general problems of a similar kind. From their work we can conclude that if $f(t)$ is a piecewise continuous bounded function then the above problem has a solution and it is unique. In addition Padmavally [3] has shown that if $f(t)$ is nondecreasing in the interval $0 \leq t \leq \tau$ then $T(0, t)$ is also nondecreasing in this interval.

Our aim is to obtain more detailed information about the surface temperature $T(0, t)$ when $f(t) \geq 0$ and $f(t)$ is integrable. First we shall obtain a sequence of upper and lower bounds on $T(x, t)$, which incidentally provide a constructive proof of its existence, and we shall also show its uniqueness. Then we shall show that as $t \rightarrow \infty, T(0, t) \sim$ $\pi^{1 / 2} E(\infty) t^{-1 / 2}$ where $E(\infty)$ is the net energy flux into the solid through the surface. Furthermore, we shall show that $E(\infty)>0$ for $n \geq 3$ while $E(\infty)=0$ for $n \leq 2$. Thus for $n \geq 3$ some of the energy which enters the solid remains there, while for $n \leq 2$ it is all ultimately radiated away. We shall also examine the behavior of $T(0, t)$ for small values of $t$ as well as for large and small values of $\alpha$.

2. Equivalent integral equation. A solution $T(x, t)$ of (1.1)-(1.4) can be represented in terms of $T(0, t)$ by the formula

$$
T(x, t)=\int_{0}^{t} f(s) G_{\rho}(x, t, s) d s
$$

* Received March 7, 1971. The research reported in this paper was supported by the Army Research Office, Durham, under Contract No. DA-31-124-ARO-D-361. 


$$
+\int_{0}^{t}\left[\rho(s)-\alpha T^{n-1}(0, s)\right] T(0, s) G_{\rho}(x, t, s) d s, \quad t \geq 0, \quad x \geq 0 .
$$

This formula is obtained by applying Green's theorem to $T(x, t)$ and the Green's function $G_{\rho}$ defined by the following linear problem:

$$
\begin{aligned}
& G_{\rho, t}=G_{\rho, x x}, \quad x>0, \quad t \geq s \geq 0, \\
& G_{\rho, x}(0, t, s)=\rho(t) G_{\rho}(0, t, s)-\delta(t-s), \quad t \geq s, \\
& G_{\rho}(x, t, s)=0, \quad t<s, \quad x \geq 0, \\
& G_{\rho}(x, t, s) \rightarrow 0 \quad \text { as } \quad x \rightarrow \infty, \quad t \geq s .
\end{aligned}
$$

The nonnegative function $\rho(t)$ in (2.3) is arbitrary, and can be chosen to facilitate the analysis. Any solution $T(x, t)$ of (2.1) satisfies (1.1)-(1.4).

We now set $x=0$ in (2.1) to obtain a nonlinear integral equation for $T(0, t)$ :

$$
\begin{aligned}
T(0, t)= & \int_{0}^{t} f(s) G_{\rho}(0, t, s) d s \\
& +\int_{0}^{t}\left[\rho(s)-\alpha T^{n-1}(0, s)\right] T^{\prime}(0, s) G_{\rho}(0, t, s) d s, \quad t \geq 0 .
\end{aligned}
$$

Once $T(0, t)$ is found from (2.6), it can be used in (2.1) to yield a solution $T(x, t)$ of (1.1)-(1.4). Thus the problem is reduced to solving (2.6).

Let us denote by $u_{\rho}(x, t)$ the first term on the right side of $(2.1)$, i.e.

$$
u_{\rho}(x, t)=\int_{0}^{t} f(s) G_{\rho}(x, t, s) d s .
$$

It is evident that $u_{\rho}$ is the solution of the linear problem (2.2)-(2.5) with $\delta(t-s)$ replaced by $f(t)$. Now (2.6) can be written in the form

$$
T(0, t)=u_{\rho}(0, t)+\int_{0}^{t}\left[\rho(s)-\alpha T^{n-1}(0, s)\right] T(0, s) G_{\rho}(0, t, s) d s .
$$

When $\rho(t) \equiv 0,(2.6)$ and (2.8) become the following simple-looking equation:

$$
T(0, t)=\pi^{-1 / 2} \int_{0}^{t}\left[f(s)-\alpha T^{n}(0, s)\right](t-s)^{-1 / 2} d s .
$$

3. Bounds on $T(x, t)$. Let us define the sequences of functions $u_{i}$ and $\rho_{i}$ as follows:

$$
\begin{aligned}
u_{j}(x, t) & =u_{\rho_{i}}(x, t), \quad j=1,2, \cdots, \\
\rho_{0}(t) & \equiv 0, \quad \rho_{j}(t)=\alpha\left[u_{i-1}(0, t)\right]^{n-1}, \quad j=1,2, \cdots .
\end{aligned}
$$

By the maximum principle, $G_{\rho} \geq 0$ and then from (2.7) and the assumption that $f \geq 0$ we have $u_{i} \geq 0$. Now for any two functions $\rho(t)$ and $\bar{\rho}(t)$, the functions $u_{\rho}$ and $u_{\bar{\rho}}$ given by (2.7) are related by the integral equation

$$
u_{\bar{\beta}}(x, t)=u_{\rho}(x, t)+\int_{0}^{t}[\rho(s)-\bar{\rho}(s)] u_{\bar{\beta}}(0, s) G_{\rho}(x, t, s) d s .
$$

From (3.2) it follows first that $u_{1} \leq u_{1}$ and then that $u_{1} \leq u_{2} \leq u_{0}$. By induction we 
find

$$
0 \leq u_{1} \leq u_{3} \leq \cdots \leq u_{2 j-1} \leq \cdots \leq u_{2 j} \leq \cdots \leq u_{2} \leq u_{0}, x \geq 0, \quad t \geq 0
$$

The functions $u_{2 i-1}$ form a monotone increasing sequence bounded above by $u_{0}$, while the $u_{2}$ form a monotone decreasing sequence bounded below by zero. Thus both sequences converge to limits, $u^{0}$ and $u^{e}$, defined by

$$
\lim _{j \rightarrow \infty} u_{2 j-1}=u^{0}, \quad \lim _{j \rightarrow \infty} u_{2 j}=u^{e} \text {. }
$$

By using (3.2) in a suitable way, we can show that $u^{e}=u^{0}=u(x, t)$, say, and that $u(0, t)$ is the unique solution of (2.9). Furthermore, $u(x, t)$ is the unique solution of (1.1)-(1.4). (See Appendix A for details.) Thus the sequence $u_{i}(0, t)$ converges to the unique solution $T(0, t)$ of (2.9), providing a constructive proof of its existence, as was shown by Mann and Wolf [1] for a different sequence. From (3.4) and (3.3) it follows that the $u_{2 j-1}$ form an increasing sequence of lower bounds on $T(x, t)$ while the $u_{2 i}$ form a decreasing sequence of upper bounds:

$0 \leq u_{1} \leq u_{3} \leq \cdots \leq u_{2 i-1} \leq \cdots \leq T \leq \cdots \leq u_{2 i} \leq \cdots \leq u_{2} \leq u_{0}, \quad x \geq 0, t \geq 0$,

In particular, (3.5) yields $T(x, t) \geq 0$.

Another interesting lower bound on $T(0, t)$ can be obtained by choosing $\rho(t)=\rho^{*}(t)$ in (2.3) where

$$
\rho^{*}(t)=\alpha M t^{-1}, \quad t>0, \quad M>0 .
$$

In Appendix B we show that as $t \rightarrow \infty$,

$$
u_{\rho^{*}}(0, t) \sim C^{*} t^{-1 / 2}, \quad C^{*}>0 .
$$

We now use $\rho^{*}$ and $u_{\rho}$ in (2.8) to obtain

$T(0, t)=u_{\rho^{*}}(0, t)+\alpha \int_{0}^{t}\left\{M s^{-1}-[T(0, s)]^{n-1}\right\} T(0, s) G_{\rho^{*}}(0, t, s) d s, \quad t \geq 0$.

Now $T(0, t)$ is positive, bounded, and decays at least as fast as $t^{-1 / 2}$ as $t \rightarrow \infty$, as we see from (3.5) and (4.8). Therefore it is possible to choose $M$ so large that $M t^{-1}-[T(0, t)]^{n-1}$ $\geq 0$ for all $t>0$ provided that $n \geq 3$. Then it follows from (3.8) and (3.7) that

$$
T(0, t) \geq u_{\rho *}(0, t) \sim C^{*} t^{-1 / 2}, \quad C^{*}>0, \quad n \geq 3 .
$$

We now assume that $0 \leq f(t) \leq C$ where $C>0$. Then we define $\mu$ and $K$ by

$$
\mu=\alpha n K^{n-1}, \quad K=(C / \alpha)^{1 / n} \text {. }
$$

Upon setting $\rho=\mu$ in (2.8), we obtain

$$
\begin{aligned}
T(0, t)= & u_{\mu}(0, t)+\alpha(n-1) K^{n} \int_{0}^{t} G_{\mu}(0, t, s) d s \\
& -\alpha \int_{0}^{t}\left[(n-1) K^{n}-n K^{n-1} T(0, s)+T^{n}(0, s)\right] G_{\mu}(0, t, s) d s .
\end{aligned}
$$

In (3.11) we use the easily proved inequality $(n-1) K^{n}-n K^{n-1} T+T^{n} \geq 0$ if $n \geq 1$, $T \geq 0, K \geq 0$. We also use the fact stated above that $G_{\mu} \geq 0$, and then (3.11) yields 


$$
\begin{aligned}
T(0, t) \leq u_{\mu}(0, t)+\alpha(n-1) & K^{n} \int_{0}^{t} G_{\mu}(0, t, s) d s \\
& \leq\left[C+\alpha(n-1) K^{n}\right] \int_{0}^{t} G_{\mu}(0, t, s) d s, \quad n \geq 1 .
\end{aligned}
$$

In Appendix $\mathrm{C}$ we show that the integral in (3.12) is bounded above by $\mu^{-1}$, so (3.12) becomes

$$
T(0, t) \leq K=(C / \alpha)^{1 / n}, \quad n \geq 1 .
$$

To obtain another lower bound we define $\gamma$ by

$$
\gamma=\alpha K^{n-1}=\alpha^{1 / n} C^{1-1 / n}
$$

Then we set $\rho=\gamma$ in (2.8) and then use (3.13) to obtain

$T(0, t)=u_{\gamma}(0, t)$

$$
+\alpha \int_{0}^{t}\left\{K^{n-1}-[T(0, s)]^{n-1}\right\} T(0, s) G_{\gamma}(0, t, s) d s \geq u_{\gamma}(0, t), \quad n \geq 1 .
$$

The lower bound $u_{\gamma}$ in (3.15) is given by (2.7). For any constant $\gamma>0, G_{\gamma}$ is given by

$$
G_{\gamma}(0, t, s)=\pi^{-1}(t-s)^{-1 / 2} \int_{0}^{\infty} \frac{\xi^{1 / 2} e^{-\xi}}{\xi+\gamma^{2}(t-s)} d \xi, \quad t>s, \quad \gamma \geq 0 .
$$

We now use (3.16) in (2.7) and evaluate $u_{\gamma}$ for $t$ large. Then (3.15) yields

$$
T(0, t) \geq u_{\gamma}(0, t) \sim C_{\gamma} t^{-3 / 2}, \quad C_{\gamma}>0, \quad n \geq 1 .
$$

4. Behavior of $T(0, t)$ for $t \rightarrow \infty$. By integrating (1.1) with respect to $x$ from 0 to $\infty$ and with respect to $t$ from 0 to $t$ and using (1.2)-(1.4), we obtain

$$
\int_{0}^{t}\left[f(s)-\alpha T^{n}(0, s)\right] d s=\int_{0}^{\infty} T(x, t) d x .
$$

The left side of (4.1) is $E(t)$, the net energy flow into the solid up to time $t$, while the right side is the energy in the solid at time $t$. We have shown above that if $f \geq 0$ then $T(x, t) \geq 0$, and thus the right side of (4.1) is nonnegative. Therefore (4.1) yields

$$
E(t) \equiv \int_{0}^{t}\left[f(s)-\alpha T^{n}(0, s)\right] d s \geq 0 \text { if } f \geq 0 .
$$

From (4.2) we obtain

$$
\int_{0}^{\infty} T^{n}(0, s) d s<\infty \quad \text { if } \int_{0}^{\infty} f(s) d s<\infty .
$$

We can now determine the behavior of $T(0, t)$ for $t \rightarrow \infty$ by utilizing (4.3) to evaluate the integral in (2.9) asymptotically. We see at once that

$$
T(0, t) \sim \pi^{-1 / 2} \int_{0}^{\infty}\left[f(s)-\alpha T^{n}(0, s)\right] d s t^{-1 / 2} \sim \pi^{-1 / 2} E(\infty) t^{-1 / 2} .
$$

Upon using (4.4) in (3.9) we obtain

$$
E(\infty) \geq \pi^{1 / 2} C^{*}>0, \quad n \geq 3 .
$$


By using (4.4) in (4.3), we see that when $E(\infty)>0$ the integral of $T^{n}$ is finite only if $n>2$. It follows that

$$
E(\infty)=0, \quad n \leq 2 .
$$

Thus (4.4) shows only that $T(0, t)=o\left(t^{-1 / 2}\right)$ for $n \leq 2$. On the other hand, (3.17) shows that $T(0, t)$ does not decrease faster than $t^{-3 / 2}$ for $n \geq 1$.

When $n=1$ the explicit solution of (2.8) is

$$
T(0, t)=u_{\alpha}(0, t) \sim C_{\alpha} t^{-3 / 2}, \quad C_{\alpha}>0, \quad n=1, \quad \alpha>0 .
$$

Thus for $n=1, T(0, t)$ decays at the fastest rate permitted by (3.17). However if $\alpha=0$, which we have hitherto excluded, then (2.9) shows that $T(0, t)$ is independent of $n$ and is given by

$$
T(0, t)=u_{0}(0, t) \sim C_{0} t^{-1 / 2}, \quad C_{0}>0, \quad \alpha=0 .
$$

Comparison of (4.4) with (4.8) shows that for $n>2, T(0, t)$ decays at the same slow rate $O\left(t^{-1 / 2}\right)$ as if the boundary were not radiating. To understand this we write the radiation rate $\alpha T^{n}$ as $\alpha^{\prime}(t) T$ with the effective radiation constant $\alpha^{\prime}(t)=\alpha T^{n-1}$. Now for $n>1, \alpha^{\prime}(t)$ tends to zero as $t \rightarrow \infty$, so the boundary tends to behave as a nonradiating boundary $(\alpha=0)$ as $t \rightarrow \infty$. Evidently for $1<n<2, \alpha^{\prime}(t)$ does not tend to zero fast enough to make $T(0, t)$ decay as slowly as $t^{-1 / 2}$, but for $n>2$ it does.

5. Perturbation expansions. To find $T(0, t)$ for small values of $\alpha$, we use (2.9) and solve it by iterations. For $\alpha$ small we can write the results as

$$
\begin{aligned}
T(0, t)= & u_{0}(0, t)-\alpha \pi^{-1 / 2} \int_{0}^{t} \frac{u_{0}^{n}(0, s)}{(t-s)^{1 / 2}} d s \\
& +n \alpha^{2} \pi^{-1} \int_{0}^{t} \frac{u_{0}^{n-1}(0, s)}{(t-s)^{1 / 2}} \int_{0}^{s} \frac{u_{0}^{n}(0, r)}{(s-r)^{1 / 2}} d r d s+O\left(\alpha^{3}\right) .
\end{aligned}
$$

For $t$ small, we require $f(t)$ to be such that $u_{0}(0, t)$ has the expansion

$$
u_{0}(0, t)=a t^{h}+b t^{a}+0\left(t^{a}\right), \quad t \rightarrow 0, \quad q>h .
$$

Then the iterative solution of (2.9) yields

$$
\begin{aligned}
T(0, t)= & a t^{h}+b t^{a}+0\left(t^{a}\right)-\alpha \pi^{-1 / 2} a^{n} \Gamma_{n h} t^{n h+1 / 2}\left[1+0\left(t^{\alpha-h}\right)\right] \\
& +\alpha^{2} n \pi^{-1} a^{2 n-1} I_{n h} I_{2 n h-h+1 / 2} t^{(2 n-1) h+1}\left[1+0\left(t^{\alpha-h}\right)\right], \quad t \rightarrow 0 .
\end{aligned}
$$

Here we have introduced $I_{d}$, defined by

$$
I_{d}=\int_{0}^{1} \frac{s^{d}}{(1-s)^{1 / 2}} d s
$$

To find $T(0, t)$ for $\alpha$ large, we first use the Abel inversion formula to solve (2.9) for $T^{n}$ in the form

$$
T^{n}(0, t)=\frac{f(t)}{\alpha}-\frac{1}{\alpha \pi^{1 / 2}} \frac{d}{d t} \int_{0}^{t}(t-s)^{1 / 2} T(0, s) d s
$$


Then we iterate (5.5) to obtain

$$
\begin{aligned}
T(0, t)= & \alpha^{-1 / n}[f(t)]^{1 / n} \\
& -\alpha^{-2 / n} n^{-1} \pi^{-1 / 2}[f(t)]^{1 / n-1} \frac{d}{d t} \int_{0}^{t}[f(s)]^{1 / n}(t-s)^{-1 / 2} d s+O\left(\alpha^{-3 / n}\right), \quad t>0
\end{aligned}
$$

The result (5.6) cannot be valid at $t=0$ because $f(0)$ may not be zero, whereas $T(0,0)$ must be zero. It is not valid for $t$ large if $f(t)$ decays too fast. Thus an initial layer expansion is required at and near $t=0$, and another expansion may be needed for large $t$, but we shall not determine it.

Appendix A. Existence and uniqueness. To show that $u^{c} \equiv u^{0}$, we consider (3.2) with $\rho(t)=\alpha\left[u_{2 j}(0, t)\right]^{n-1}$ and $\bar{\rho}(t)=\alpha\left[u_{2 j-1}(0, t)\right]^{n-1}$. Then taking limits as $j \rightarrow \infty$ yields the equation

$$
u^{e}(x, t)-u^{0}(x, t)=\int_{0}^{t}\left[u^{e}(0, s)-u^{0}(0, s)\right] \Re(x, t, s) d s, \quad t \geq 0, \quad x \geq 0,
$$

where

$$
\Re(x, t, s)=\frac{\left[u^{e}(0, s)\right]^{n-1}-\left[u^{0}(0, s)\right]^{n-1}}{u^{e}(0, s)-u^{0}(0, s)} u^{\bullet}(0, s) G_{p}(x, t, s) \geq 0 .
$$

By setting $x=0$ in (A.1) we obtain

$$
u^{e}(0, t)-u^{0}(0, t)=\int_{0}^{t}\left[u^{\bullet}(0, s)-u^{0}(0, s)\right] \Re(0, t, s) d s, \quad t \geq 0 .
$$

This can be viewed as a homogeneous integral equation of the second kind for $u^{e}(0, t)-$ $u^{0}(0, t)$ with $\Re(0, t, s)$ as the kernel. If we choose a $t$ such that $\left|u^{e}(0, s)-u^{0}(0, s)\right| \leq$ $\left|u^{e}(0, t)-u^{0}(0, t)\right|$ for $0 \leq s \leq t$, then (A.3) yields

$$
\left|u^{\bullet}(0, t)-u^{0}(0, t)\right| \leq\left|u^{e}(0, t)-u^{0}(0, t)\right| \int_{0}^{t} \Re(0, t, s) d s .
$$

For $t$ sufficiently small, say $0 \leq t \leq \epsilon$, the integral in (A.4) is less than unity, which implies that $u^{\circ}(0, t)=u^{0}(0, t)$ for $t \leq \epsilon$. Using this fact in (A.3), we can show that $u^{e}(0, t)=u^{0}(0, t)$ in a larger interval. This procedure can be repeated to show that $u^{c}(0, t)=u^{0}(0, t)$ for all $t \geq 0$. Then (A.1) shows that $u^{e}(x, t)=u^{0}(x, t)$ for all $x \geq 0$, $t \geq 0$. Thus there is a common limit $u(x, t)$, so

$$
u(x, t)=u^{e}(x, t)=u^{0}(x, t), \quad x \geq 0, \quad t \geq 0 .
$$

It follows from the definition (3.1) of $u_{i}$ and from (3.2) that $u$ and $u_{i-1}$ satisfy

$$
u_{j}(x, t)=u_{\rho}(x, t)+\int_{0}^{t}\left\{\rho(s)-\alpha\left[u_{i-1}(0, s)\right]^{n-1}\right\} u_{i}(0, s)\left(j_{\rho}(x, t, s) d s .\right.
$$

Then since $u_{i} \rightarrow u$ and $u_{i-1} \rightarrow u$, it is clear from (A.6) that $u$ satisfies (2.1).

To show that the nonnegative solution constructed above is unique, we assume that there are two solutions $T_{1}$ and $T_{2}$. By subtracting (2.9) for $T_{2}$ from (2.9) for $T_{1}$ we obtain 


$$
\begin{aligned}
T_{1}(0, t)- & T_{2}(0, t) \\
& =\pi^{-1 / 2} \alpha \int_{0}^{t}\left[T_{1}(0, s)-T_{2}(0, s)\right]\left\{\frac{T_{1}^{n}(0, s)-T_{2}^{n}(0, s)}{T_{1}(0, s)-T_{2}(0, s)}(t-s)^{-1 / 2}\right\} d s .
\end{aligned}
$$

Now by the same arguments used above to show that $u^{e}(0, t)=u^{0}(0, t)$, it follows that $T_{1}(0, t)=T_{2}(0, t)$. Then from (2.1) it follows that $T_{1}(x, t)=T_{2}(x, t)$.

Appendix B. Asymptotic behavior of $u_{\rho^{*}}(0, t)$. Tor establish the asymptotic proerty (3.7) for $u_{\bullet} \cdot(0, t)$, we consider the initial boundary value problem (2.2)-(2.5) for $u_{\rho_{*} *}$ with $\rho(t)=\rho^{*}(t)=\alpha M t^{-1}$ and with $\delta(t-s)$ replaced by $f(t)$. Applying the Laplace transform to this problem yields

$$
\begin{gathered}
\hat{u}_{\bullet^{* x x}}(x, p)+p \hat{u}_{\rho^{*}}(x, p)=0, \quad x>0, \\
\hat{u}_{\rho^{\bullet . .}}(0, p)=\alpha M \int_{0}^{\infty} e^{-p t} t^{-1} u_{\rho^{*}}(0, t) d t-\hat{f}(p), \\
\hat{u}_{\rho^{*}}(x, p) \rightarrow 0, \quad x \rightarrow \infty .
\end{gathered}
$$

Here $\hat{u}_{\rho^{*}}(x, p)$ and $\hat{f}(p)$ are defined by

$$
\hat{u}_{\rho}(x, p)=\int_{0}^{\infty} e^{-p t} u_{\rho}(x, t) d t, \quad \hat{f}(p)=\int_{0}^{\infty} e^{-p t} f(t) d t .
$$

The solution of (B.1) satisfying (B.3) is

$$
\hat{u}_{p *}(x, p)=A(p) e^{-p^{2 / 2 x}} .
$$

Here $A(p)=\hat{u}_{\rho^{*}}(0, p)$ must be determined from the boundary condition (B.2). Upon substitution of (B.5) into (B.2) we obtain

$$
-p^{1 / 2} A(p)=\alpha M \int_{0}^{\infty} e^{-p t} t^{-1} u_{p^{*}}(0, t) d t-\hat{f}(p) .
$$

Differentiation of (B.6) with respect to $p$ yields

$$
-\frac{d}{d p}\left[p^{1 / 2} A(p)\right]=-\alpha M A(p)-\frac{d}{d p} \hat{f}(p) .
$$

The solution of (B.7) which satisfies (B.6) is

$$
A(p)=-p^{-1 / 2} \exp \left[2 \alpha M p^{1 / 2}\right] \int_{D}^{\infty} \exp \left[-2 \alpha M \xi^{1 / 2}\right] \hat{f}^{\prime}(\xi) d \xi .
$$

As $p \rightarrow 0$, (B.8) implies that

$$
A(p) \sim p^{-1 / 2} \int_{0}^{\infty} \exp \left[-2 \alpha M \xi^{1 / 2}\right] \int_{0}^{\infty} t f(t) e^{-\xi t} d t d \xi \text { as } p \rightarrow 0 .
$$

Then a classical asymptotic result on Laplace transforms shows that

$$
u_{\rho^{*}}(0, t) \sim C t^{-1 / 2} \text { as } t \rightarrow \infty, \quad C>0 .
$$

Appendix C. Estimation of an integral. To estimate the integral in (3.12) we consider (2.2)-(2.5) with $\rho(t)=\mu=$ constant. Upon integrating the differential equa- 
tion (2.2) we obtain

$\int_{0^{-}}^{t^{+}} \int_{0}^{\infty} G_{\mu, t}(x, t, s) d s d x=\int_{0^{-}}^{t^{+}} \int_{0}^{\infty} G_{\mu, x x}(x, t, s) d s d x=-\int_{0^{-}}^{t^{+}} G_{\mu, x}(0, t, s) d s$

By virtue of the boundary condition (2.3) we then have

$$
\int_{0-}^{t^{+}} \int_{0}^{\infty} G_{\mu, t}(x, t, s) d s d x=1-\mu \int_{0}^{t} G_{\mu}(0, t, s) d s .
$$

Since $G_{\mu}(x, t, s)$ depends on the difference $t-s, G_{\mu, t}=-G_{\mu, \text { and }}$ (C.2) becomes

$$
0 \leq \int_{0}^{\infty} G_{\mu}^{\prime}(x, t, 0) d x=1-\mu \int_{0}^{t} G_{\mu}(0, t, s) d s, \quad t>0 .
$$

This gives the desired inequality

$$
\int_{0}^{t} G_{\mu}(0, t, s) d s \leq \mu^{-1}
$$

\section{REFERENCES}

[1] W. R. Mann and F. Wolf, Heat transfer between solids and gases under nonlinear boundary conditions, Quart. Appl. Math. 9, 163-184 (1951)

[2] J. H. Roberts and W. R. Mann, A certain nonlinear integral equation of the Volterra type, Pacific J. Math. 1, 431-445 (1951)

[3] K. Padmavally, On a nonlinear integral equation, J. Math. Mech. 7, 533-555 (1958)

[4] A. Friedman, Generalized heat transfer between solids and gases under nonlinear boundary conditions, J. Math. Mech. 8, 161-183 (1959) 